\title{
Couplage d'un modèle de neige avec un modèle de climat
}

\author{
par Eric Brun, Eric Martin \\ Centre d'Etudes de la Neige, Météo-France/CNRM
}

Valery Spiridonov (*)

National Institute of Meteorology and Hydrology, Bulgarian Academy of Science

\section{INTRODUCTION}

Le manteau neigeux joue un rôle très important dans le climat de la Terre car la neige a des propriétés physiques très particulières. Le manteau neigeux est en continuelle évolution sous l'effet des conditions météorologiques. La métamorphose des cristaux de neige est le principal responsable de cette évolution qui est très rapide et susceptible de modifier les propriétés physiques de la neige de plus d'un ordre de grandeur. Cela entraîne une modification sensible des échanges d'énergie et de masse entre la neige et l'atmosphère.

Pour ces raisons, des paramétrisations de la neige et des modèles de neige spécifiques ont été développés pour améliorer la prise en compte des effets dus au manteau neigeux dans les modèles de climat (GCM) [1, 2, 3, 4]. Ces modèles ne simulent pas la métamorphose de la neige, ce qui ne per- met pas de calculer l'albédo de la neige de façon réaliste, c'est-à-dire en fonction du type et de la taille des cristaux à sa surface $[5,6]$.

Plutôt que de développer un nouveau modèle de neige pour les GCM capable de simuler la métamorphose, nous avons préféré utiliser le modèle de neige Crocus et le coupler au GCM Arpege. En effet Crocus est un modèle de neige numérique multicouche développé pour la prévision du risque d'avalanches et qui simule la plupart des processus internes au manteau neigeux en utilisant des méthodes basées sur la physique : métamorphose, percolation de l'eau liquide, dégel/regel, tassement et conduction thermique. Le couplage avec le GCM est synchrone et basé sur un échange des flux entre la surface de la neige et la base de l'atmosphère à chaque pas de temps.

(*) Étude conduite à GMAP, Météo-France/CNRM, Toulouse.

\section{Coupling a multi-layered snow model with a GCM}

A multi-layered snow model, including most physical processes governing the evolution of snowpacks, has been coupled to a GCM to improve the representation of snow cover in climate simulations. The snow model, called Crocus, includes original features to simulate the evolution of snowpack layering which allows a realistic calculation of snow albedo as a function of the type and size of the crystals of the surface layer. The coupling scheme is based on a synchronous run of the GCM and of the snow model with an exchange of the surface fluxes at every time step. It was tested in a 5 year run at a T42 resolution. The impact on the atmosphere was important over most covered regions and the snowpacks simulated in the different regions present a layering which is realistic and very variable in connection with the climate. The simulated snow cover compares satisfactorily with the present snow climatology. 


\section{II — LE MODÈLE DE NEIGE CROCUS}

Crocus est un modèle de neige numérique multicouche et unidimensionnel développé pour l'étude de la neige et la prévision du risque d'avalanches. Il diffère des autres modèles de neige développés pour l'hydrologie ou l'étude de la neige $[7,8,9,10,11,12]$ par son aptitude à simuler la stratification du manteau neigeux. Nous entendons par là le calcul de l'évolution de la taille et du type de cristaux composant chacune des couches de neige constituant le manteau neigeux. Les cristaux sont décrits selon un formalisme original qui a une correspondance possible avec la Classification Internationale de la Neige [13]. L'évolution des cristaux se fait par l'intermédiaire d'un ensemble de paramétrisations décrivant l'ensemble des lois de métamorphose : faible, moyen et fort gradient de température pour la neige sèche et métamorphose de neige humide. Les principaux processus pris en compte dans Crocus sont les suivants :

- diffusion de chaleur par le biais d'un coefficient de conduction effective fonction de la densité de la neige et de la température qui prend en compte la diffusion de vapeur due au gradient de température,

- percolation de l'eau liquide lorsque la teneur en eau liquide dépasse la valeur en eau de rétention de la neige exprimée comme fonction du volume des pores,

- fonte et regel dans les couches internes et superficielles du manteau neigeux,

- tassement en considérant la neige comme un fluide Newtonien de viscosité dépendant de la température, de la densité, du type et de la taille des cristaux de neige,

- absorption du rayonnement solaire en surface et en profondeur en utilisant un albédo et un coefficient d'absorption fonction de la longueur d'onde ( 3 bandes spectrales), de la taille et du type des cristaux ainsi que de l'âge des cristaux à la surface (pour représenter l'effet de salissement par dépôt sec ou humide).

Crocus comprend jusqu'à 50 couches numériques dont le nombre et l'épaisseur varient à chaque pas de temps de façon à accorder couches numériques et couches stratigraphiques. Une description détaillée de Crocus est présentée dans les publications $[14,15]$.

Le modèle de neige Crocus a été testé dans des contextes d'utilisation variés à l'échelle locale et régionale et l'on peut considérer qu'il fait partie des modèles de neige les plus validés. A l'échelle locale, la validation a été faite sur le site du Col de Porte (Chartreuse, $1320 \mathrm{~m}$ ), notamment durant l'hiver 1988-1989. A partir des données météorologiques mesurées sur le site (température et humidité de l'air, vitesse du vent, rayonnement solaire et rayonnement thermique incidents, précipitations neigeuses et pluvieuses), le modèle a simulé correctement et tout au long de la saison les principales caractéristiques observées du manteau neigeux : hauteur de neige, température de surface, écoulement à la base, profil de température, de teneur en eau liquide et de densité ainsi que la stratigraphie [15].

A l'échelle régionale, la validation a tout d'abord été conduite en simulant 10 années d'évolution du manteau neigeux sur 37 points de mesure répartis dans les Alpes françaises entre 900 et $3000 \mathrm{~m}$ d'altitude [16]. Les données météorologiques nécessaires en entrée de Crocus n'étaient évidemment pas mesurées sur ces sites et elles furent fournies par le modèle d'analyse météorologique Safran qui utilise tout type d'observation météorologique disponible dans les Alpes et dans les régions environnantes [17]. L'analyse de ces données ne fut pas faite sur les 37 points de contrôle mais sur les différents massifs des Alpes auxquels ces points sont rattachés. Ce sont des zones climatologiquement homogènes d'une superficie de $1000 \mathrm{~km}^{2}$ environ. Les données d'entrée de Crocus n'étaient donc pas disponibles à l'échelle locale mais à l'échelle régionale. Malgré l'incertitude sur ces données, inhérente à la méthode d'analyse, la hauteur de neige simulée par le modèle sans calibration locale était très satisfaisante sur la plupart des points, excepté pour les sites fortement soumis au transport de neige par le vent ou les sites où les conditions météorologiques locales diffèrent trop de celles du reste du massif (fond de vallées encaissées par ex.).

Une seconde validation à l'échelle régionale a été menée par le biais du couplage entre le modèle hydrologique ETH/HBV et Crocus sur un bassin versant alpin de $224 \mathrm{~km}^{2}$ [18]. Dans ce couplage, le modèle hydrologique utilisait comme données d'entrée les lames d'eau liquide simulées par Crocus à la base du manteau neigeux ou analysées par Safran en cas de sol non enneigé. Ces valeurs calculées par tranche d'altitude de $200 \mathrm{~m}$ et pour différentes expositions des pentes étaient pondérées par les surfaces relatives de ces pentes vis-à-vis de la surface totale du bassin versant. Les débits quotidiens de la rivière ont été simulés sur une période de 10 ans et comparés aux débits mesurés par EDF/DTG. Les résultats obtenus ont été bons et de qualité comparable à celle des débits simulés par la version classique de ETH/HBV qui calcule la fonte avec une méthode par index calibrée.

\section{III — SCHÉMA DE COUPLAGE ENTRE LE MODÈLE DE NEIGE ET LE GCM}

Nous avons travaillé avec un modèle atmosphérique spectral nommé Arpege qui est utilisé aussi bien pour la prévision météorologique opérationnelle que pour l'étude du climat. Arpege a été développé conjointement par le GMAP (Météo-France/CNRM) et par le CEPMMT sous le nom d'Arpege-IFS $[19,20]$. Une version à aire limitée a été développée sous le nom d'Aladin.

\subsection{Schéma de couplage}

Arpege calcule les échanges d'énergie et de masse entre l'atmosphère et les surfaces continentales par le biais du schéma classique "force-restore " proposé par Bhumralkar [21] et [22]. La surface est représentée par une seule couche à température de surface $T_{\mathrm{s}}$ et température profonde $T_{d}$. Elle peut être recouverte partiellement de neige. Le but du couplage était de remplacer cette couche unique par une couche de sol partiellement couverte par un manteau neigeux multicouche dont l'évolution est simulée par le modèle de neige Crocus.

Dans la version classique d'Arpege, l'évolution de la température et de l'humidité des couches atmosphériques et de la surface pendant un temps $d t$ est calculée simultanément selon un schéma implicite. La relation liant l'humidité et la température superficielles est utilisée pour résoudre simultanément l'évolution de ces deux variables sur tout le profil atmosphérique et en surface. Ce schéma nécessite l'inversion d'une matrice tridiagonale composée de termes dépendant de la température et de l'humidité des couches atmosphériques et de la surface. Ce type de schéma ne peut intégrer la résolution simultanée du calcul de l'évolution de la température des couches de neige à cause des processus de gel-dégel qui font que l'évolution de la température de la neige va dépendre aussi de l'évolution de la teneur en eau liquide des couches de neige. Pour cette raison, nous avons choisi d'activer les deux modèles séparément et de façon synchrone. Le principe retenu pour le calcul incrémental de l'évolution de l'atmosphère, du sol et de la neige en un point de grille et entre les pas de temps $t$ et $t+d t$ est le suivant (illustration sur la figure 1 ) :

- 1) les propriétés physiques de la surface (capacité calorifique, conductivité thermique, rugosité, albédo, température 


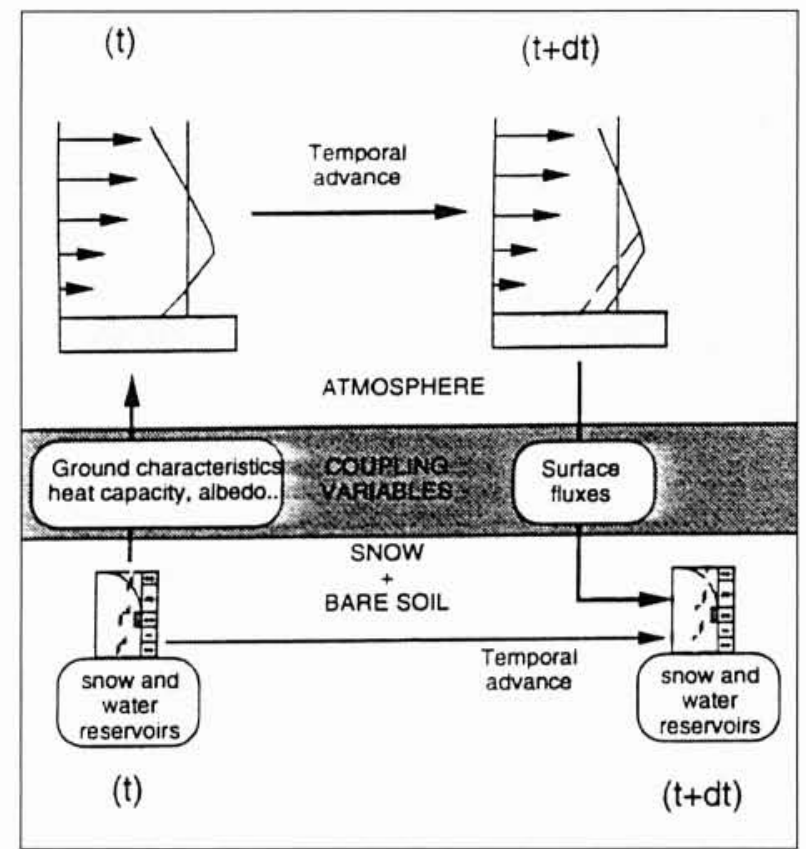

1. Description du schéma de couplage entre le modèle de neige et le modèle atmosphérique.

profonde) pendant le pas de temps $d t$ sont calculées selon une pondération des propriétés physiques respectives du sol et du manteau neigeux. La pondération est faite selon la proportion de sol enneigé pneij.

- 2) les valeurs prises par les propriétés physiques de la surface sont utilisées par le modèle atmosphérique Arpege pour calculer l'évolution de la température et de l'humidité des couches atmosphériques et de la surface.

- 3) les flux d'énergie et de vapeur d'eau échangés entre la surface et l'atmosphère entre les temps $t$ et $t+d t$ sont calculés par Arpege et transmis au modèle Crocus qui peut alors calculer l'évolution correspondante du manteau neigeux.

- 4) le taux de fonte nivale, le flux d'eau liquide s'écoulant à la base du manteau neigeux et le flux d'énergie échangé entre la base du manteau neigeux et le sol sous-jacent entre les temps $t$ et $t+d t$ sont calculés par Crocus et transmis au modèle atmosphérique Arpege qui peut alors faire évoluer son réservoir de neige et la température et l'humidité du sol.

Le schéma présenté ci-dessus est semblable aux schémas utilisés pour coupler les modèles atmosphériques aux modèles océaniques. On peut donc considérer qu'il s'agit effectivement d'un couplage qui assure une interaction totale des deux modèles sans correction de flux. L'utilisation de cette technique se fait sans modification fondamentale des modèles.

En contrepartie, le fait que le modèle de neige considère jusqu'à 50 couches de neige multiplie approximativement le coût du modèle par deux, aussi bien pour le temps de calcul que pour la mémoire utilisée. Un tel coût pourrait être facilement abaissé en limitant le nombre de couches de neige mais le but de ce travail était la prise en compte de la plupart des processus physiques pilotant l'évolution du manteau neigeux, ce qui nécessite une stratigraphie détaillée.

Aucune paramétrisation de l'interaction neige-végétation n'a été prise en compte mais la proportion de neige pneij dans une maille dépend aussi de la rugosité de la surface et donc de la végétation. Les propriétés physiques de la surface d'une maille enneigée dépendent ainsi sensiblement de la couverture végétale considérée.

\subsection{Correction des échanges turbulents de chaleur en cas très stable}

Des changements mineurs ont été nécessaires dans le modèle atmosphérique et dans le modèle de neige. Les premiers essais en mode couplé ont montré une diminution de 20 à $30^{\circ} \mathrm{C}$ de la surface en Antarctique par rapport à la version originale d'Arpege. Cela parce que la conductivité thermique de la surface calculée à partir de la densité de la neige était beaucoup plus faible, ce qui diminuait le flux de chaleur fourni par les couches de neige en profondeur et refroidissait la surface de neige. Le phénomène était grandement amplifié par une diminution irréaliste des flux de chaleur turbulents, liée à une augmentation de la stabilité de la couche atmosphérique superficielle. Cela était dû à la paramétrisation de ces échanges dans Arpege [23, 24] qui est peu adaptée aux forts nombres de Richardson. Nous avons résolu grossièrement ce problème en limitant le nombre de Richardson à une valeur limite de 0,5 .

\subsection{Densification de la neige par le vent}

Le second problème rencontré venait d'une sous-estimation systématique de la densité de la neige de surface en région polaire. En effet, Crocus calcule un taux de tassement basé sur une viscosité Newtonienne dépendant de la température, de la densité et du type de neige. La prédominance de températures très froides en surface limitait fortement le tassement. En Antarctique, la densité de surface est fréquemment plus forte que $200 \mathrm{~kg} \mathrm{~m}^{-3}$ et atteint souvent $300 \mathrm{~kg} \mathrm{~m}^{-3}$, bien qu'en région alpine cette densité soit typiquement de $100 \mathrm{~kg} \mathrm{~m}^{-3}$ et souvent beaucoup plus faible lors de chutes de neige froide non ventées. La forte densité observée en Antarctique est le plus souvent due au transport de neige par le vent. C'est pourquoi nous avons développé une paramétrisation originale pour simuler les effets du transport de neige sur la densification. Elle est basée sur le modèle de Guyomarc'h et al. [25] qui a été établi à partir d'observations sur le terrain en région alpine.

Pour chaque maille du modèle, nous calculons en fonction du type de neige simulé un indice de mobilité et un seuil de vent au dessus duquel le transport se déclenche. Par exemple, le seuil pour la neige fraîche (dendricité égale à 1) à une densité de $50 \mathrm{~kg} \mathrm{~m}^{-3}$ est de $2,8 \mathrm{~m} \mathrm{~s}^{-1}$. Si en un point de grille, le vent excède le seuil, nous considérons que le transport se produit, ce qui induit une densification et une modification de la neige simulée en ce point de grille. Son efficacité sur la neige de surface est exprimée comme une fonction de la force du vent et de la mobilité de la neige superficielle. L'efficacité sur les couches plus profondes décroît selon une fonction exponentielle de la profondeur et de la mobilité des couches supérieures. A titre d'exemple, $10 \mathrm{~cm}$ sous la surface dans une couche de neige fraîche peu dense, l'efficacité du transport sur la métamorphose et sur la densification est divisée par $e$. Cette paramétrisation permet de compacter rapidement la neige des régions froides mais ventées. Elle agit non seulement pendant la chute de neige elle-même mais parfois aussi quelques jours ou quelques semaines après la chute selon l'évolution que le manteau neigeux a subie entre temps.

\subsection{Test des modèles couplés}

Le couplage du modèle de neige Crocus avec le GCM Arpege fut d'abord testé avec la version aire limitée du modèle atmosphérique appelée Aladin. Avec des points de grille distants de $15 \mathrm{~km}$ environ, l'intérêt de ce test portait sur l'analyse de la stabilité numérique du schéma de couplage. Le modèle fut activé sur une période d'un mois 
s'étendant de la fin décembre 1994 à la fin janvier 1995 sur une zone couvrant l'Europe occidentale et centrale. Aucune instabilité ne fut observée sans modification du pas de temps originel fixé à $120 \mathrm{~s}$. Dans ce test, peu d'effets sur l'atmosphère ont été induits par l'utilisation du modèle de neige à la place de la paramétrisation simple utilisée dans la version classique d'Aladin qui considère une couche unique de neige différant du sol par son seul albédo. La différence la plus importante portait sur l'extension du manteau neigeux.

Le test principal fut conduit en activant les modèles couplés à l'échelle globale sur une simulation de 5 ans, à une résolution T42 et avec 31 niveaux verticaux dans l'atmosphère. L'évolution de la température océanique superficielle et de la glace de mer était forcée par une climatologie mensuelle (AMIP 1979-1988). Dans cet article, nous nous focalisons principalement sur la simulation du manteau neigeux et sur la climatologie nivale plutôt que sur l'impact du couplage sur la circulation atmosphérique.

\subsection{Les manteaux neigeux simulés}

L'originalité de Crocus vient du fait qu'il simule l'évolution stratigraphique du manteau neigeux. Comme il n'existe pas dans la littérature de description des caractéristiques du manteau neigeux à l'échelle globale, il est difficile de valider les profils stratigraphiques simulés par le modèle couplé. Nous nous contenterons donc de décrire quelques-uns de ces profils qui mettent en évidence l'aptitude du modèle à simuler des manteaux neigeux très variables en liaison avec les caractéristiques climatiques locales. La figure 2 montre l'évolution durant une saison de 4 manteaux neigeux situés à la même latitude. Le code couleur correspond aux différents types de neige [15]. Les hachures représentent les couches humidifiées puis regelées (croûtes). Le premier manteau neigeux simulé est situé dans le SW de la Sibérie $\left(62^{\circ} \mathrm{N}, 60^{\circ} \mathrm{E}\right.$, $204 \mathrm{~m}$ ). L'enneigement permanent commence en octobre et subit l'humidification jusqu'en décembre. Ensuite, de faibles chutes de neige et la prédominance du froid le rendent sec avec formation de givre de profondeur jusqu'à la mi-mars quand une première humidification temporaire affecte la neige de surface. Une humidification totale se produit en mai, suivie d'un regel complet. La fonte s'achève à la fin mai. Le deuxième manteau neigeux est situé en Sibérie orientale $\left(62^{\circ} \mathrm{N}, 138^{\circ} \mathrm{E}, 1035 \mathrm{~m}\right)$ dans une région soumise à un de climats les plus continentaux et les plus froids de l'Hémisphère Nord. Le manteau neigeux commence en septembre et reste sec jusqu'au début juin. Il fond alors très rapidement, en deux semaines seulement. Le troisième manteau neigeux se situe en Alaska dans la région d'Anchorage $\left(62^{\circ} \mathrm{N}, 132^{\circ} \mathrm{W}, 1279 \mathrm{~m}\right)$. Il differre fortement des deux précédents. L'accumulation est très forte et commence en octobre. L'humidification persiste jusqu'à la fin novembre. Un manteau neigeux sec composé principalement de grains fins est simulé jusqu'à la mi-mai. La fonte dure approximativement deux mois. Cette région est réputée pour ses manteaux neigeux épais et relativement denses bien reproduits par la simulation. Le dernier manteau neigeux simulé se situe dans la partie Sud du Groenland $\left(62^{\circ} \mathrm{N}, 48^{\circ} \mathrm{W}, 642 \mathrm{~m}\right)$. Il est caractérisé par des chutes de neige très précoces en sep-
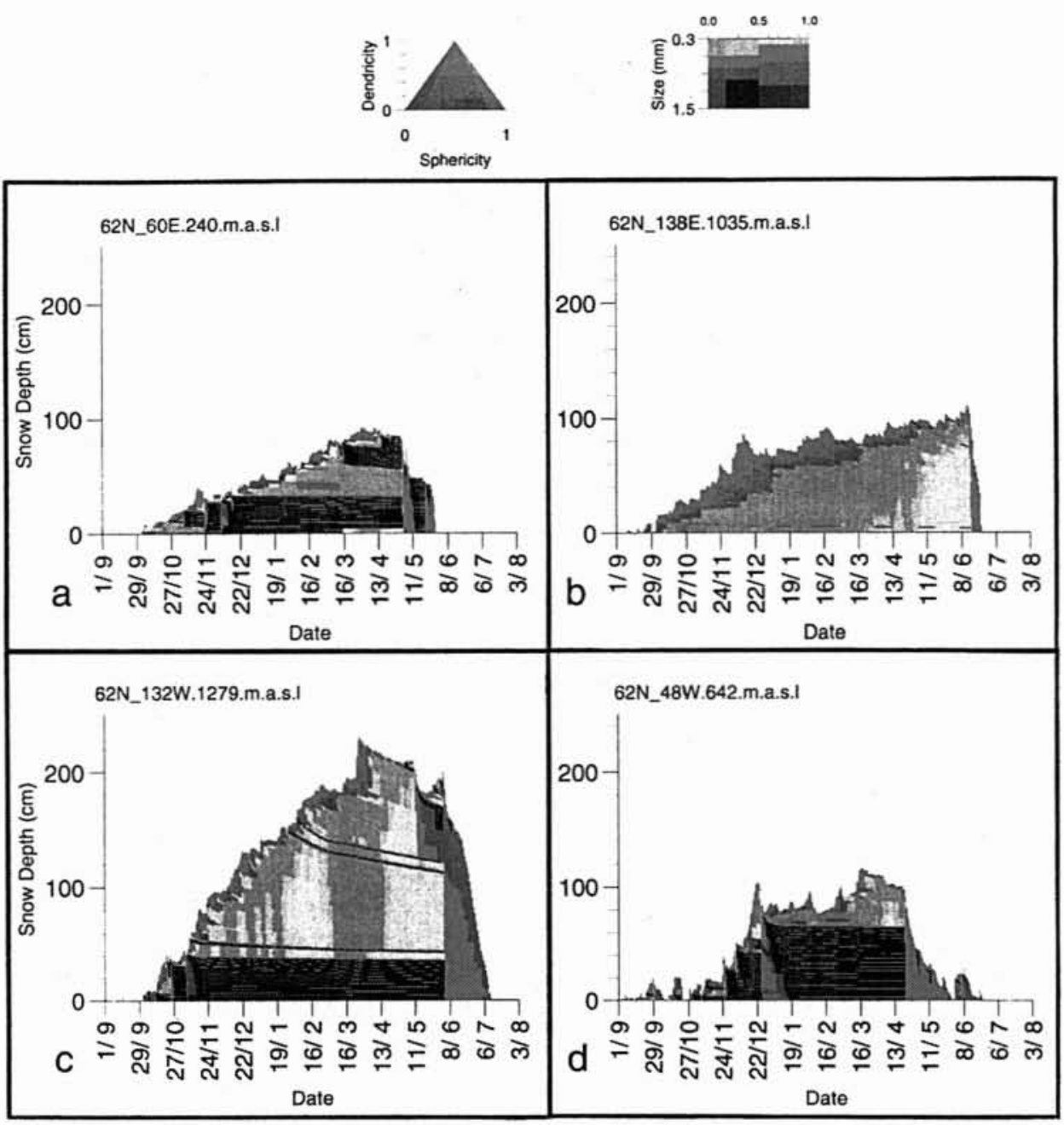

2. Simulation du manteau neigeux en 4 points du globe à la même latitude. 


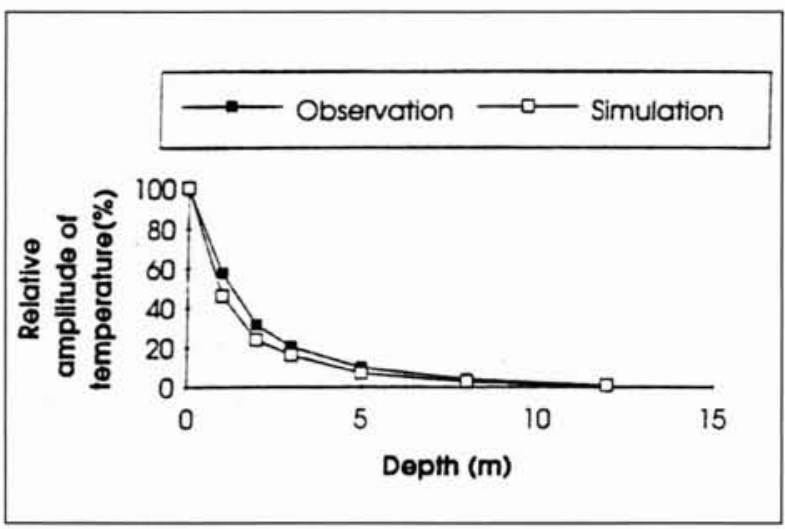

3. Comparaison entre simulation et observation de l'atténuation de l'amplitude thermique dans le névé au Pôle Sud.

tembre qui subissent humidification et parfois fonte totale jusqu'à la fin décembre. Cela est dû à l'influence des perturbations maritimes qui prédominent en automne dans cette région. Un manteau neigeux sec et froid est simulé de janvier à mi-avril suivi par une longue période de fonte lente et partielle interrompue par de nouvelles chutes de neige jusqu'à la mi-juin. A la fin janvier, on observe sur cette simulation un événement de transport de neige important qui a densifié une trentaine de $\mathrm{cm}$ de neige tombée un mois auparavant.

Bien que les manteaux neigeux décrits plus haut soient localisés à la même latitude, leurs caractéristiques differrent très sensiblement, ce qui montre la capacité du modèle de neige couplé au GCM à simuler des manteaux très variables en liaison avec les conditions climatiques prédominantes.

Crocus peut simuler des couches superficielles de neige de très faible densité, ce qui induit une faible capacité thermique et aussi une très faible conductivité. Cela diminue sensiblement le flux de chaleur des couches profondes. Pour illustrer avec quel réalisme ce phénomène est modélisé, nous avons comparé sur la figure 3 l'atténuation avec la profondeur de l'amplitude annuelle de la température dans la neige simulée au Pôle Sud. Pour cela, nous avons utilisé des mesures faites en 1958 et les 5 ans de simulation du profil de température de la neige au point $\left(88^{\circ} \mathrm{S}, 90^{\circ} \mathrm{E}, 3137 \mathrm{~m}\right)$. Comme dans les observations, la température à $10 \mathrm{~m}$ sous la surface est presque constante et très proche de la moyenne annuelle de la température de surface $\left(-52{ }^{\circ} \mathrm{C}\right.$ pour la simulation et $-51^{\circ} \mathrm{C}$ pour l'observation). La légère sous-estimation de l'amplitude des ondes de température en profondeur est due à la sous-estimation persistante de la densité de surface malgré la prise en compte des effets du transport de neige (typiquement $270 \mathrm{~kg} \mathrm{~m}^{-3}$ au lieu de $370 \mathrm{~kg} \mathrm{~m}^{-3}$ en surface).

La durée du manteau neigeux dans les différentes régions du globe est une composante importante du climat. La figure 4 compare la fréquence d'enneigement durant 4 périodes de l'année simulée par les modèles couplés et calculée par Groisman et al. [27]. Dans la plupart des régions de l'hémisphère Nord, le modèle simule une climatologie de l'enneigement très proche de la réalité. Les plus grandes différences se produisent là où le relief du GCM à la résolution T42 diffère le plus du relief réel (les Alpes et les Montagnes Rocheuses dans leur partie Sud par exemple). Dans de tels cas, la différence d'altitude entre les points de grille correspondants et la réalité ne permet pas de simulation correcte de l'enneigement.
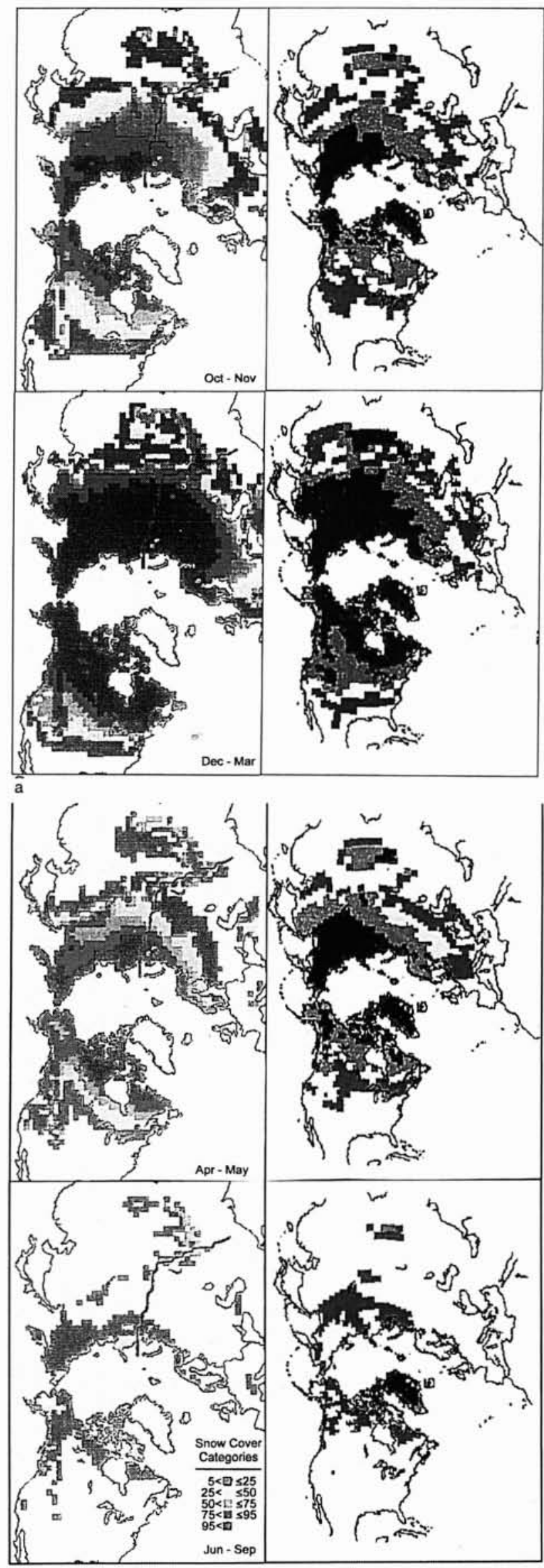

4. Comparaison entre fréquence saisonnière d'enseignement simulée et observée (en partie tirée de [27]). 


\subsection{Impact sur le climat}

Simuler dans un modèle atmosphérique l'évolution du manteau neigeux avec un modèle sophistiqué au lieu d'une paramétrisation plus simple change significativement les échanges d'énergie entre le manteau neigeux et l'atmosphère. C'est dû non seulement à un changement de l'albédo de la neige mais aussi à un changement des propriétés thermiques du manteau neigeux, réduisant sensiblement le flux de chaleur du sol profond, notamment au cœur de l'hiver. Globalement, la comparaison entre modèles couplés et simulation de référence (modèle dans sa version classique) ne présente de modifications sensibles du climat que dans les régions enneigées. Cela est illustré sur la figure 5 qui compare la température simulée et observée en Antarctique durant le mois de juillet. La température de l'air simulée avec le modèle couplé sur le plateau antarctique est plus froide et plus réaliste que celle simulée avec le modèle de référence. Comme cette région est complètement couverte de neige dans les deux simulations et comme la nuit y est presque permanente en juillet, ceci met en évidence que la différence entre les résultats est due dans ce cas précis aux seules modifications des propriétés thermiques de la neige. Celles-ci ont donc une importance comparable aux propriétés radiatives. En moyenne annuelle, l'albédo du plateau antarctique est plus élevé avec le modèle couplé qu'avec le modèle classique $(0,85$ au lieu de 0,80$)$.

Etant donné qu'aucune attention particulière n'a été accordée dans cette étude à l'interaction neige-végétation, aucune amélioration n'est attendue sur le climat des régions enneigées couvertes de forêts qui représentent pourtant la plus grande partie des régions enneigées de l'hémisphère nord.
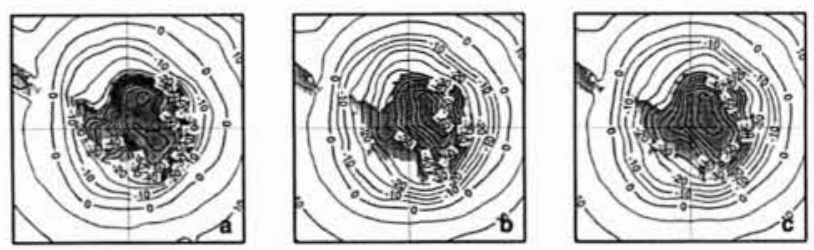

5. Comparaison entre température de l'air $2 \mathrm{~m}$ au dessus de la surface antarctique analysée par le CEPMMT (a), simulée par le modèle couplé (b) et par le modèle atmosphérique dans sa version d'origine.

\section{IV $\square$ CONCLUSION}

Le couplage d'un GCM avec un modèle de neige multicouche permet de simuler l'évolution du manteau neigeux lors de simulations climatiques. Les manteaux neigeux simulés dans les différentes régions présentent des caractéristiques réalistes et l'impact du couplage est important sur la climatologie nivale et le climat des régions enneigées. Le schéma de couplage choisi dans cette étude assure une interaction totale entre le manteau neigeux et l'atmosphère. Cependant, le nombre important de couches de neige considérées (jusqu'à 50 pour les manteaux épais et fortement stratifiés) accroît fortement le coût des simulations climatiques. Ce n'est donc intéressant que pour les études climatiques focalisées sur les processus cryosphériques. Nous avons montré qu'il est possible de simuler le climat des régions polaires tout en simulant la température interne du névé. Une simulation réaliste de la conductivité thermique des différentes couches du manteau neigeux offre des perspectives intéressantes pour l'étude du permafrost. Naturellement, le couplage est aussi très intéressant pour l'étude des impacts possibles d'un changement climatique sur le manteau nei- geux tout en considérant les effets de feed-back. La principale limitation du développement présenté dans ce papier vient de l'absence d'une paramétrisation spécifique pour la prise en compte des interactions neige-atmosphère.

\section{REMERCIEMENTS}

Les auteurs remercient chaleureusement Jean-François Geleyn (Météo-France/GMAP) pour ses précieux conseils dans la conception du schéma de couplage, Michel Déqué et Alain Braun (Météo-France/GMGEC) pour leur aide efficace dans l'activation de la version climatique du modèle Arpege ainsi que Hervé Giordani (Météo-France/GMGEC) qui nous a aidés à utiliser le simulateur 1-D de la physique d'Arpege. Nous remercions aussi Eric Guilyardi qui nous a fourni le logiciel graphique Vairmer utilisé pour réaliser la figure 5 .

\section{RÉFÉRENCES}

[1] Douville H., Royer J.F. and MAhfouf J.F. (1995a). - A new snow parametrization for the Météo-France climate model. Part I. Validation in stand-alone experiments. Climate Dyn., 12(1), 21-35.

[2] Douville H., Royer J.F. and Mahfouf J.F. (1995b), - A new snow parametrization for the Météo-France climate model. Part II. Validation in a 3-D GCM experiment. Climate Dyn., 12(1), 37-52.

[3] Loth B., Graf H.F. and Oberhuber J.M. (1993). - Snow cover model for global climate simulations. J. Geophys. Res.,98(D6), 10,451-10, 464.

[4] LYNCH-STIEgLrTz M. (1994). - The development and validation of a simple snow model for GISS GCM. J. Climate, 7(12), 1842-1855.

[5] Wiscombe W.J. and WARREN S.G. (1980). - A model for the spectral albedo of snow. I. Pure snow. J. Atmos. Sci., 37(12), 2712-2733.

[6] Sergent C., Pougatch E. and Sudul M. and Bourdelles B. (1993), - Experimental investigation of optical snow properties. Ann. Glaciol., 17, 281-287.

[7] OBLed C. (1971). - Modèle mathématique de la fonte nivale. (Thèse de Doctorat, Université Scientifique et Médicale de Grenoble.)

[8] Navarre J.P. (1975). - Modèle unidimensionnel d'évolution de la neige déposée : modèle perce-neige. La Météorologie, 4(3), 103-120.

[9] ANDERSon E.A. (1976). - A point energy and mass balance model for a snow cover. NOAA Tech. Rep. NWS-19.

[10] JORDAN R. (1991). - A one-dimensional temperature model for a snow cover: technical documentation for SNTHERM.89. CRREL Spec. Rep. 91-16.

[11] Bader H.P. and Weilenmann P. (1992). - Modeling temperature distribution, energy and mass flow in a (phase changing) snowpack. I Model and Case studies. Cold Reg. Sci. Technol., 20(2), 157-181.

[12] Yamazaki T., Kondo J., Sakuraokoa T. and Nakamura T. (1993). - A one dimensional model of the evolution of snow-cover characteristics. Ann. Glaciol. 18, 22-26.

[13] COLBECK S.C. and 7 others. (1990). - The international classification for seasonal snow on the ground. Boulder, CO, International Commission on Snow and Ice and World Data Center A for Glaciology. 
[14] Brun E., Martin E., Simon V., Gendre C. and Coléou C. (1989). - An energy and mass model of snow cover suitable for operational avalanche forecasting. J. Glaciol., 35 (121), 333342.

[15] Brun E., David P., Sudul M. and Brunot G. (1992). A numerical model to simulate snow-cover stratigraphy for operational avalanche forecasting. J. Glaciol., 38 (128), 13-22.

[16] Martin E., Brun E. and Durand Y. (1994). - Sensitivity of the French Alps snow cover to the variation of climatic variables. Annales Geophysicae, Ser. Atmospheres, Hydrospheres and Space Sciences, 12(5), 469-477.

[17] Durand Y., Brun E., Merindol L., Guyomarc'h G., LESAFrRE B. and MARTIN E. (1993). - A meteorological estimation of relevant parameters for snow models. Ann. Glaciol., $18,65-71$.

[18] Braun L.N., Brun E., Durand Y., Martin E. and TouRASSE P. (1994), - Simulation of discharge using different methods of meteorological data distribution, basin discretization and snow modelling. Nord. Hydrol.,25(1-2) 129-144.

[19] Courtier P. and Geleyn J.F. (1988), - A global numerical weather prediction model with variable resolution : application to shallow-water equations. Q. J. R. Meteorol. Soc., 114(483), 1321-1346.

[20] Déqué M., Devreton C., Braun A. and Cariolle D. (1994). - The ARPEGE/IFS atmosphere model : a contribution to the French community climate modelling. Climate Dyn., 10(4/5), 249-266.
[21] BhUMRALKaR C.M. (1975). - Numerical experiments on the computation of ground surface temperature in atmospheric general circulation model. J. Appl. Meteorol., 14 (7), 12461258 .

[22] BlackadAR A.K. (1976). - Modeling the nocturnal boundary layer. in Third Symposium on Atmospheric Turbulence, Diffusion and Air Quality. Proceedings. Boston, MA,American Meteorological Society, 46-49.

[23] Louss J.F. (1979). - A parametric model of vertical eddy fluxes in the atmosphere. Boundary-Layer Meteorol., 17(2), 187-202.

[24] Musson-Genon L. (1995). - Comparison of different turbulence closures with a one-dimensional boundary layer model. Mon. Wea. Rev., 123(1), 163-180.

[25] Guyomarc'h G., Mérindol. L., Castelle T., Sivardière F. and Buisson L. (1994). - Blowing snow and avalanches. Proceedings, International Snow Science Workshop, 30 October-3 November 1994, Snowbird, Utah, 211-221.

[26] Dalrymple P.C., Lettau H.H. and Wollaston S.H. (1966), - South Pole micrometeorology program : data analy sis. In Rubin, M.J., ed. Studies in Antarctic meteorology. Washington, DC, American Geophysical Union, 13-56 (Antarctic Research Series 9).

[27] Groisman P.Ya., Karl. T.R., KNight R.W. and StenchiKov G.L. (1994). - Changes of snow cover, temperature and radiative heat balance over the Northern Hemisphere. J. Climate, 7(11), 1633-1656. 